\title{
A Study on Transmission Loss Characteristics of Honeycomb Panel for Offshore Structures
}

\author{
Jae-Deok Jung1, Suk-Yoon Hong1, Jee-Hun Song'2, Hyun-Woung Kwon ${ }^{3}$ \\ ${ }^{1}$ Department of Naval Architecture and Ocean Engineering, Seoul National University, Seoul, Republic of Korea \\ ${ }^{2}$ Department of Naval Architecture and Ocean Engineering, Chonnam National University, Yeosu, Republic of \\ Korea \\ ${ }^{3}$ Department of Naval Architecture and Ocean Engineering, Koje College, Koje, Republic of Korea \\ Email: jaedeok@snu.ac.kr, syh@snu.ac.kr, jhs@jnu.ac.kr, khw1@koje.ac.kr
}

Received 19 November 2014

\begin{abstract}
Honeycomb panel is consisted of 3 layers that are double-faced sheets and honeycomb-shaped core. It is highly desirable for ship, railway, and aerospace industry. The reason is that honeycomb panel excels in strength and in its weight. However in terms of insulation, it is a little bit insufficient to commonly use sandwich-panel. In this paper, Moor's theory is used to predict sound transmission loss (STL). The theory is assumed that core layer is homogeneous orthotropic. And to calculate STL, it is evaluated in terms of the symmetric and anti-symmetric panel impedances, and the characteristic impedance of air. After that predicted data are compared with experiment data.
\end{abstract}

\section{Keywords}

\section{Honeycomb Panel, Sound Transmission Loss (STL), Orthotropic Core}

\section{Introduction}

A honeycomb panel has good mechanical properties. So the honeycomb panels are widely used in diverse industries from its low weight and high strength. In addition to this merits, we want more insulation performance. To do this we predict STL of honeycomb-panel. That is compared with experiment data.

A study of transmission loss for the unbounded orthotropic sandwich panel with honeycomb core was investigated by Wang Shengchun [1]. An analysis of the transmission loss in the sandwich panels with orthotropic cores was presented by Moore and Lyon [2].

It is used for multi-layered system later on methodology for analyzing the multi-layered system come from one general method by Brouard, Lafarge and Allard [3] [4].

\section{Theory}

Honeycomb have different stiffness moduli in planes perpendicular and parallel to the direction of the cells, and 
can be characterized as orthotropic with nine independent stiffness constants. Equation (1) is express relation of stress, strain, and stiffness constants [5].

$$
\left\{\begin{array}{l}
\sigma_{x} \\
\sigma_{y} \\
\sigma_{z} \\
\tau_{y z} \\
\tau_{x z} \\
\tau_{x y}
\end{array}\right\}=\left\{\begin{array}{cccccc}
E_{11} & E_{12} & E_{13} & 0 & 0 & 0 \\
& E_{22} & E_{23} & 0 & 0 & 0 \\
& & E_{33} & 0 & 0 & 0 \\
& & & E_{44} & 0 & 0 \\
& & & & E_{55} & 0 \\
& & & & & E_{66}
\end{array}\right\}\left\{\begin{array}{c}
\varepsilon_{x} \\
\varepsilon_{y} \\
\varepsilon_{z} \\
\gamma_{y z} \\
\gamma_{x z} \\
\gamma_{x y}
\end{array}\right\}
$$

where $\sigma_{x}, \sigma_{y}$ and $\sigma_{z}$ are the normal stresses in the $x, y$, and $z$ directions, respectively. $\tau_{y z}, \tau_{x z}, \tau_{x y}$ are the shear stresses [6] [7]. The strains are defined with respect to the particle displacements $u, v, w$ in the $x, y, z$ directions, respectively, as follows:

$$
\begin{aligned}
& \epsilon_{x}=\frac{\partial u}{\partial x}, \quad \epsilon_{y}=\frac{\partial v}{\partial y}, \quad \epsilon_{z}=\frac{\partial w}{\partial z} ; \\
& \gamma_{x y}=\frac{\partial u}{\partial y}+\frac{\partial v}{\partial x}, \quad \gamma_{x z}=\frac{\partial u}{\partial z}+\frac{\partial w}{\partial x}, \quad \gamma_{y z}=\frac{\partial v}{\partial z}+\frac{\partial w}{\partial y} .
\end{aligned}
$$

The stored elastic potential energy density, $W$, for a given strain field is:

$$
2 W=E_{11} \epsilon_{x}^{2}+2 E_{12} \epsilon_{x} \epsilon_{y}+2 E_{13} \epsilon_{x} \epsilon_{z}+E_{22} \epsilon_{y}^{2}+2 E_{23} \epsilon_{y} \epsilon_{z}+E_{33} \epsilon_{z}^{2}+E_{44} \gamma_{x y}^{2}+E_{55} \gamma_{x z}^{2}+E_{66} \gamma_{x y}^{2}
$$

The Equation (4) is total elastic potential energy that is stored in a body of finite volume is computed as the integral of the potential energy density over the volume of the body. Kinetic energies are similarly defined in terms of volume integrals as follow Equation (5)

$$
\begin{gathered}
\text { P.E. }=\int_{\text {Vol }} W \mathrm{dVol} \\
\text { K.E. }=\frac{1}{2} \int_{\text {Vol }} \rho\left(\dot{u}^{2}+\dot{v}^{2}+\dot{w}^{2}\right) \mathrm{dVol}
\end{gathered}
$$

The Equation (6) is a Lagrange's equation that is utilized to generate the system equations describing the dynamics of the sandwich panel [8].

$$
\frac{\mathrm{d}}{\mathrm{d} t}\left(\frac{\partial K E_{\mathrm{TOT}}}{\partial \dot{q}_{r}}\right)-\frac{\partial K E_{\mathrm{TOT}}}{\partial q_{r}}+\frac{\partial P E_{\mathrm{TOT}}}{\partial q_{r}}=Q_{r}, \quad r=1,2, \cdots
$$

where $q_{r}$ is the generalized displacements, $Q_{r}$ is the generalized forces per unit area. Applying Lagrange's equation to the expressions for the kinetic and potential energies for symmetric, antisymmetric motions, and then we know the symmetric and antisymmetric impedances. The acoustic transmission coefficient is defined as the ratio of the transmitted to incident acoustic intensities. It is evaluated in terms of the symmetric and antisymmetric panel impedances, and the characteristic impedance of air.

$$
\begin{gathered}
\tau(\theta, \phi)=\left|I_{\text {trans }} / I_{\text {inc }}\right|^{2}=\left|\frac{\left(\rho_{0} c_{0} / \cos \theta\right)\left(Z_{s}-Z_{a}\right)}{\left(Z_{s}+\rho_{0} c_{0} / \cos \theta\right)\left(Z_{a}+\rho_{0} c_{0} / \cos \theta\right)}\right|^{2} \\
\bar{\tau}=\frac{\int_{0}^{2 \pi} \int_{0}^{\theta_{\text {lim }}} \tau(\theta, \phi) \sin \theta \cos \theta \mathrm{d} \theta \mathrm{d} \phi}{\int_{0}^{2 \pi} \int_{0}^{\theta_{\text {lim }}} \sin \theta \cos \theta \mathrm{d} \theta \mathrm{d} \phi} \\
\mathrm{TL}=-10 \log _{10}(\bar{\tau}) \mathrm{dB}
\end{gathered}
$$

Equation (7) is the acoustic transmission coefficient, Equation (8) is the averaged transmission coefficient, and Equation (9) is the transmission loss. 


\section{Numerical Results and Discussion}

The sound transmission loss of a honeycomb panel is present in Figures 1-4. The material properties are listed in Table 1. The core of honeycomb is aluminum and the face sheet is steel. Sound Transmission Class (STC) of $15 \mathrm{~mm}$ theory and experiment is same as $21 \mathrm{~dB}$. STC of $25 \mathrm{~mm}$ theory and experiment is $21 \mathrm{~dB}, 23 \mathrm{~dB}$, respectively. STC of $30 \mathrm{~mm}$ theory and experiment is $21 \mathrm{~dB}, 23 \mathrm{~dB}$, respectively. STC of 40 mm theory and experiment is $22 \mathrm{~dB}, 24 \mathrm{~dB}$, respectively.

According to the each thickness of panel, there are some differences in frequency but theory value and experiment value can be right within $2 \mathrm{~dB}$ in other frequency excepting for a certain frequency.

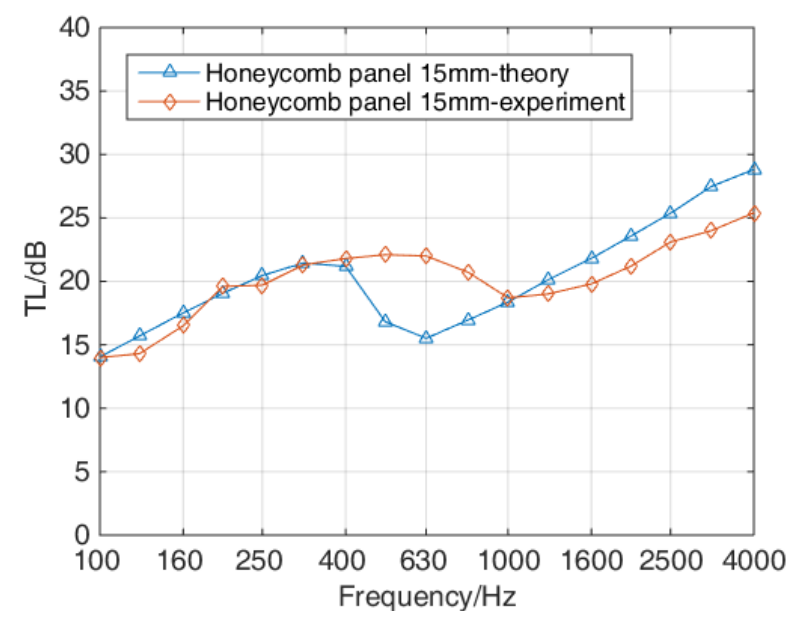

Figure 1. The STL of the honeycomb panel $15 \mathrm{~mm}$-theory and the honeycomb panel $15 \mathrm{~mm}$-experiment.

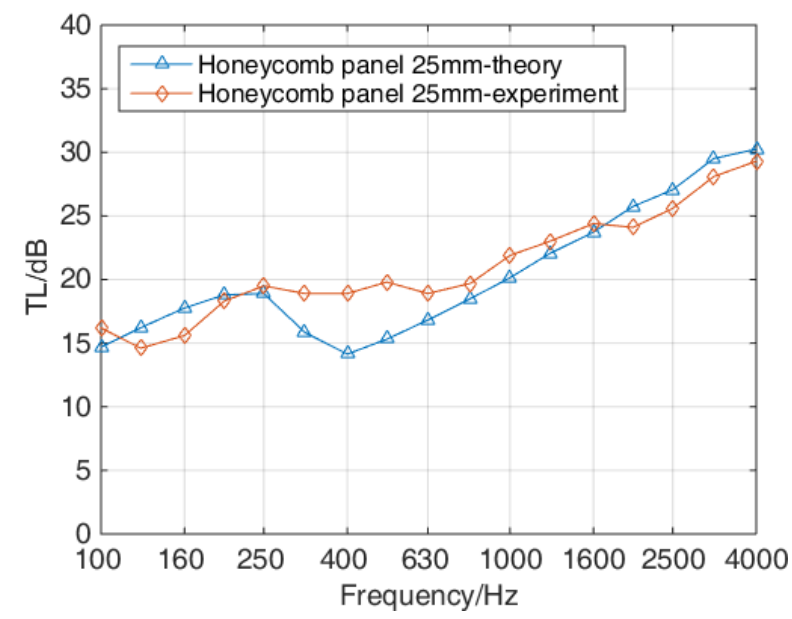

Figure 2. The STL of the honeycomb panel 25 mm-theory and the honeycomb panel $25 \mathrm{~mm}$-experiment.

Table 1. The material properties of honeycomb panel.

\begin{tabular}{ccc}
\hline Honeycomb & The face sheet & The core \\
\hline Thickness, $\mathrm{mm}$ & 0.6 & $15,25,30,40$ \\
Density, $\mathrm{kg} / \mathrm{m}^{3}$ & 7850 & 2770 \\
Young's modulus, GPa & 195 & 71 \\
Poisson coefficient & 0.3 & 0.33 \\
\hline
\end{tabular}




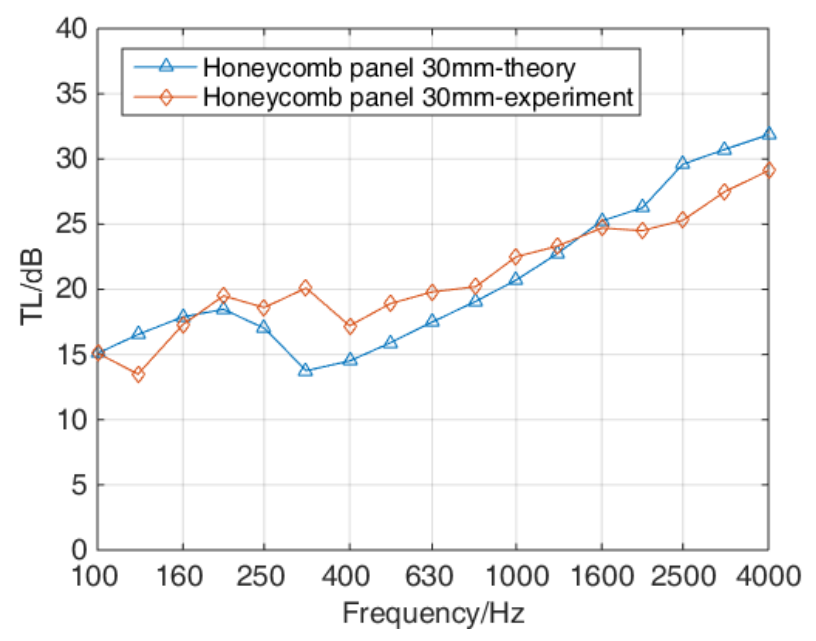

Figure 3. The STL of the honeycomb panel $30 \mathrm{~mm}$-theory and the honeycomb panel 30 mm-experiment.

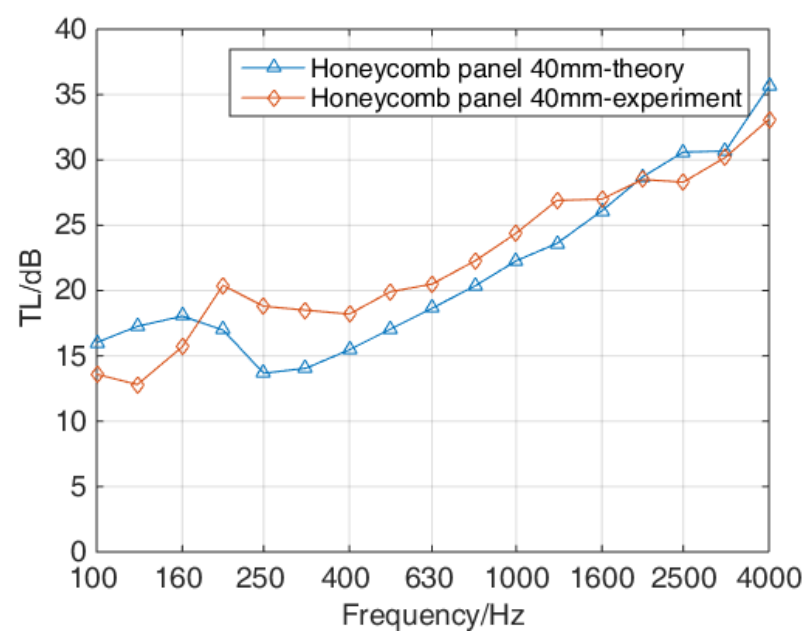

Figure 4. The STL of the honeycomb panel $40 \mathrm{~mm}$-theory and the honeycomb panel 40 mm-experiment.

\section{Conclusions}

The honeycomb panel theory is derived by Moore. Through this theory, the STL of honeycomb panel is predicted. And it is compared with experiment result according to thickness.

And then for better insulation performance, mineral wool is added to honeycomb panel. In terms of thickness both panels are same. One is a single honeycomb panel (40 t). Another is composite panel (honeycomb $15 \mathrm{~mm}+$ mineral wool $10 \mathrm{~mm}+$ honeycomb $15 \mathrm{~mm}$-theory).

This paper considers only honeycomb panel. But many elements will be studied and added on the honeycomb panel.

\section{References}

[1] Wang, S.C., Deng, Z.X. and Shen, W.D. (2010) Sound Transmission Loss Characteristics of Unbounded Orthotropic Sandwich Panels in Bending Vibration Considering Transverse Shear Deformation. Composite Structures, 92, 28852889. http://dx.doi.org/10.1016/j.compstruct.2010.04.014

[2] Moore, J.A. and Lyon, R.H. (1991) Sound Transmission Loss Characteristics of Sandwich Panel Constructions. J Acoust Soc Am, 89, 777-791.

Smith, J., Jones Jr, M., Houghton, L., et al. (1999) Future of Health Insurance. N Engl J Med, 965, 325-329. 
[3] Brouard, B., Lafarge, D. and Allard, J.-F. (1995) A General Method of Modelling Sound Propagation in Layered Media. Journal of Sound and Vibration, 183, 129-142.

Renji, K. and Nair, P.S. (1996) Modal Density of Composite Honeycomb Sandwich Panels. J Sound Vib., 195, 687699.

[4] Biot, M.A. (1956) The Theory of Propagation of Elastic Waves in a Fluid Saturated Porous Solid. The Journal of the Acoustical Society of America, 28, 168-191. http://dx.doi.org/10.1121/1.1908239

[5] Lin, H.-J., Wang, C.-N. and Kuo, Y.-M. (2007) Sound Transmission Loss across Specially Orthotropic Laminates. Applied Acoustics, 68, 1177-1191. http://dx.doi.org/10.1016/j.apacoust.2006.06.007

[6] Paik, J.K., Thayamballi, A.K. and Kim, G.S. (1999) The Strength Characteristics of Aluminum Honeycomb Sandwich Panels. Thin-Walled Structures, 35, 205-231. http://dx.doi.org/10.1016/S0263-8231(99)00026-9

[7] Kim, H.-G. and Choi, N.-S. (2004) Prediction of Mechanical Properties of Honeycomb Core Materials and Analysis of Interlaminar Stress of Honeycomb Sandwich Composite Plate. Composites Research, 17, 29-37.

[8] Moore, J. (1975) Sound Transmission Loss Characteristics of Three Layer Composite Wall Constructions. Massachusetts Institute of Technology. 